\title{
Conservative tREATMENT OF HydROTHORAX IN A PATIENT RECEIVING PERITONEAL DIALYSIS
}

Singapore Med J 2017; 58(11): 674-675 doi: 10.11622/smedj.2017102

Dear Sir,

We herein describe a novel way to prevent hydrothorax relapse in a peritoneal dialysis (PD) patient with the use of inclination, and therefore the force of gravity, to prevent peritoneal fluid from entering the thorax.

A 28-year-old woman who had been receiving automated peritoneal dialysis (APD) for two years contacted our institution's PD clinic with complaints of cough and dyspnoea. Her past medical history was significant for mental retardation, congenital upper limb impairment, end-stage renal disease due to glomerulonephritis of unknown origin, hypertension and hyperuricaemia. Clinical examination revealed oedema of the lower extremities and reduced auscultation sounds in the right lower lung that were suggestive of pleural effusion. Residual diuresis (about 1,000 mL/day) was present. Chest radiography confirmed right pleural effusion (Fig. 1a), but the patient did not consent to a diagnostic tap. Hypoalbuminaemia, cardiac failure, peritonitis and traumatic chest injury were not present.

As no further diagnostic procedures were performed, the patient's regular PD regimen, consisting of two exchanges overnight of $1.36 \%$ and $2.27 \%$ glucose dialysates in two $2.5 \mathrm{~L}$ bags, was discontinued and the patient was closely monitored. Four days after the $\mathrm{PD}$ regimen was discontinued, the peritoneal cavity remained free and reduction of pleural fluid was noted (Fig. $1 \mathrm{~b}$ ). In order to maintain adequate dialysis, the same APD prescription was resumed, but it was applied during the day and while the patient was in a sitting position. The peritoneal cavity remained free of fluid. After a month of this new regime, complete remission of symptoms was observed and chest radiography showed no pleural effusion in the right lower lung (Fig. 1c). Henceforth, the patient continued to perform APD in a sitting position during the day for another five months in order to avoid a relapse of pleural effusion. After the five-month period, overnight exchanges were started while the patient slept at a $30^{\circ}$ inclined position. There was no clinical or radiographic recurrence of the effusion 16 months later, and the patient remained on the renal replacement therapy of her choice.
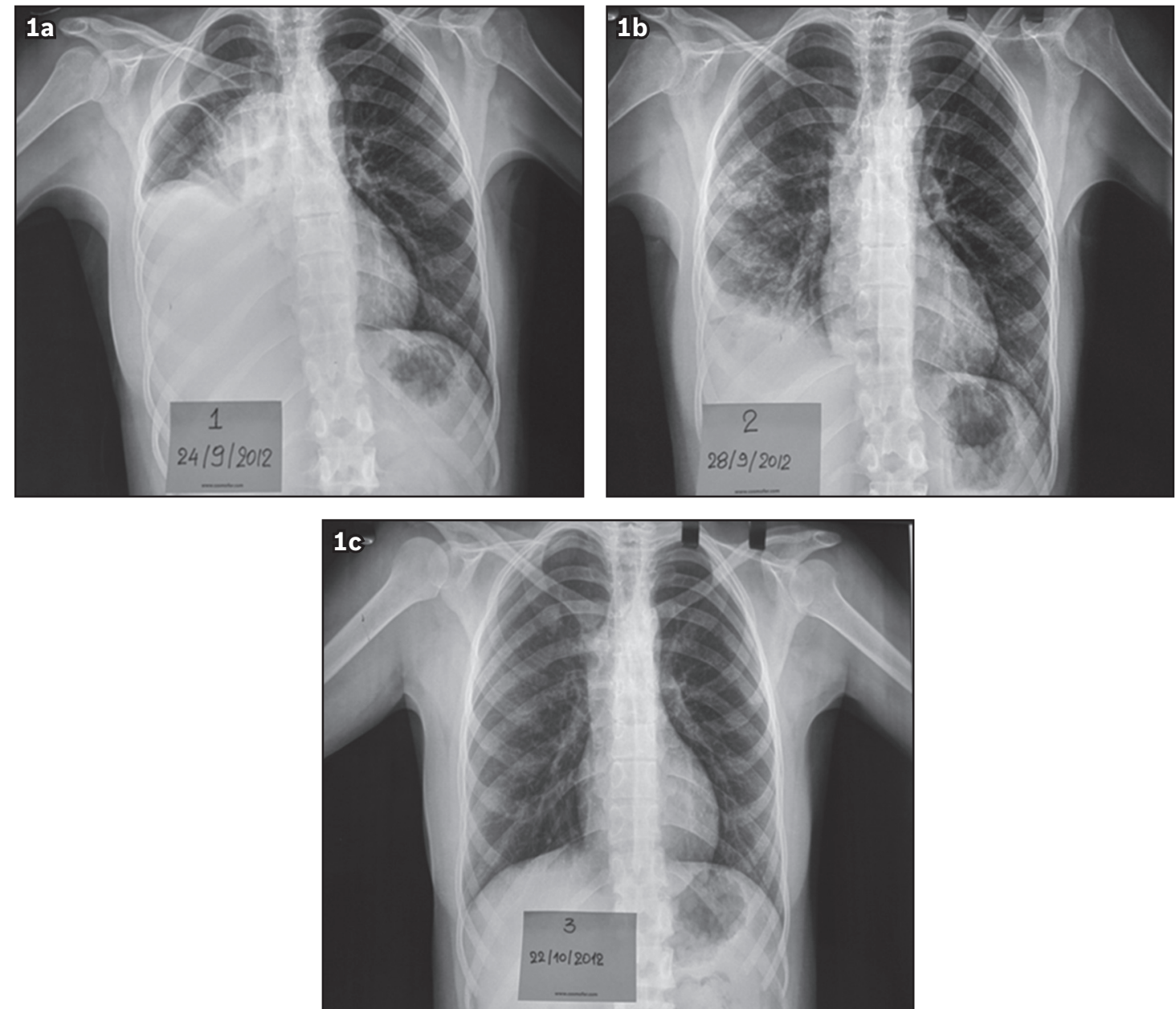

Fig. 1 (a) Initial chest radiograph shows right pleural effusion in a 28-year-old female patient who was receiving automated peritoneal dialysis (APD). (b) First follow-up chest radiograph four days after APD interruption shows reduction of pleural effusion. (c) Second follow-up chest radiograph one month later shows complete resolution of pleural effusion during the time when the patient was performing APD in a sitting position. 
Unfortunately, we were unable to document whether the patient's hydrothorax was the 'sweet' hydrothorax of PD, because the patient did not consent to further diagnostic or therapeutic interventions. Technetium-99m peritoneal scintigraphy would have been an appropriate modality to document this condition, ${ }^{(1)}$ but it was not applied. In our case, the patient's pleural effusion was attributed to fluid movement from the peritoneal cavity into the thorax due to transient increased intra-abdominal pressure. Performing PD in an initial sitting position and then in an inclined position was the best option for preventing dialysate reinsertion into the thorax, provided that adequate PD was maintained.

Open or laparoscopic surgery ${ }^{(2)}$ is the common practice for repairing the diaphragmatic defect once pleuroperitoneal communication is confirmed. However, surgical techniques are known to lead to high PD dropout rates and consequent transfers to haemodialysis, ${ }^{(3)}$ and our patient was not in favour of changing to another renal replacement therapy. In a series of children with hydrothorax, no surgical intervention was applied in the context of PD. ${ }^{(3)}$ Similar to our case, PD was performed either in a seated position or while the patient was propped upright, but with initial low volume exchanges.

In conclusion, we have described a case of PD hydrothorax that was effectively managed by interrupting the initial PD and then resuming the same PD prescription with the patient in a seated and recumbent position. Acute hydrothorax should not be a sufficient reason to discontinue PD. We recommend that other, non-surgical interventions should be attempted first.

Yours sincerely,

Chrysoula Leonidas $\underline{\text { Pipili }}{ }^{1}$, Eva Nikolaos $\underline{\text { Kiousi }}^{1}$, Edmond Periklis $\underline{\text { Ntenta }}^{1}$, Kyriaki loannis $\underline{\text { Vasileiou }}^{1}$, Eirini Ioannis $\underline{\text { Grapsa }}^{1}$ ${ }^{1}$ Department of Nephrology, Aretaieion University Hospital, Athens, Greece. chrysapi2001@gmail.com

\section{References}

1. Szeto CC, Chow KM. Pathogenesis and management of hydrothorax complicating peritoneal dialysis. Curr Opin Pulm Med 2004; 10:315-9.

2. Lew SQ. Hydrothorax: pleural effusion associated with peritoneal dialysis. Perit Dial Int 2010; 30:13-8.

3. Krishnan RG, Ognjanovic MV, Crosier J, Coulthard MG. Acute hydrothorax complicating peritoneal dialysis. Perit Dial Int 2007; 27:296-9. 